OPEN ACCESS

Edited by:

Chengdao $\mathrm{Li}$,

Murdoch University, Australia

Reviewed by:

Qing-Yong Yang,

Huazhong Agricultural University,

China

Yongqing Jiao,

Oil Crops Research Institute (CAAS),

China

Hanwei Mei,

Shanghai Agrobiological Gene Center,

China

*Correspondence:

Shaoging $L$

shaoqingli@whu.edu.cn

Specialty section:

This article was submitted to Crop Science and Horticulture,

a section of the journal

Frontiers in Plant Science

Received: 13 February 2017 Accepted: 24 May 2017

Published: 07 June 2017

Citation:

Fan F, Li N, Chen Y, Liu X, Sun H, Wang J, He G, Zhu Y and Li S (2017) Development of Elite BPH-Resistant

Wide-Spectrum Restorer Lines for

Three and Two Line Hybrid Rice.

Front. Plant Sci. 8:986.

doi: 10.3389/fpls.2017.00986

\section{Development of Elite BPH-Resistant Wide-Spectrum Restorer Lines for Three and Two Line Hybrid Rice}

\author{
Fengfeng Fan ${ }^{1}$, Nengwu Li ${ }^{1}$, Yunping Chen ${ }^{1}$, Xingdan Liu ${ }^{2}$, Heng Sun ${ }^{1}$, Jie Wang ${ }^{1}$, \\ Guangcun $\mathrm{He}^{1}$, Yingguo $\mathrm{Zhu}{ }^{1}$ and Shaoqing $\mathrm{Li}^{1 *}$
}

'State Key Laboratory of Hybrid Rice, Key Laboratory for Research and Utilization of Heterosis in Indica Rice of Ministry of Agriculture, Engineering Research Center for Plant Biotechnology and Germplasm Utilization of Ministry of Education, College of Life Science, Wuhan University, Wuhan, China, ${ }^{2}$ College of Agronomy, Hunan Agricultural University, Changsha, China

Hybrid rice has contributed significantly to the world food security. Breeding of elite high-yield, strong-resistant broad-spectrum restorer line is an important strategy for hybrid rice in commercial breeding programs. Here, we developed three elite brown planthopper (BPH)-resistant wide-spectrum restorer lines by pyramiding big-panicle gene Gn8.1, BPH-resistant genes Bph6 and Bph9, fertility restorer genes Rf3, Rf4, $R f 5$, and Rf6 through molecular marker assisted selection. Resistance analysis revealed that the newly developed restorer lines showed stronger BPH-resistance than any of the single-gene donor parent Luoyang-6 and Luoyang-9. Moreover, the three new restorer lines had broad spectrum recovery capabilities for Honglian CMS, Wild abortive CMS and two-line GMS sterile lines, and higher grain yields than that of the recurrent parent 9,311 under nature field conditions. Importantly, the hybrid crosses also showed good performance for grain yield and BPH-resistance. Thus, the development of elite $\mathrm{BPH}$-resistant wide-spectrum restorer lines has a promising future for breeding of broad spectrum BPH-resistant high-yield varieties.

Keywords: brown planthopper, fertility restorers, hybrid rice, cytoplasmic male sterility, photo-thermo-sensitive genic male sterility, molecular marker selection

\section{BACKGROUND}

The world food security is a huge challenge for human. Hybrid rice has contributed significantly to the world food production in the last decades, due to its great yield advantage over the inbred lines (Weng et al., 2008). It has been proved that heterosis utilization is one of the most effective measures to ensure food security around the world. Generally, hybrid rice can be categorized into two types, two-line based on photo-thermo-sensitive genic male sterility (GMS) and three-line based on cytoplasmic male sterility (CMS). Of which, three-line hybrid rice mainly includes Honglian (HL), Baotai (BT), and Wild abortive (WA) types based on genetic characters of cytoplasmic male sterility used for commercial production (Li et al., 2007). Unlike the two-line hybrid rice, restorer lines for three-line hybrid rice have to carry fertility restorer $(R f)$ genes that are genetically specific to some CMS type and different from each other (Li et al., 2005, 2007), which greatly limit the exploitation of heterosis because of the specific restoring-maintaining relationship. Today, there are about six $R f$ genes having been identified in rice, of which $R f 3$ and $R f 4$ for WA-CMS (Suresh et al., 2012; Luo et al., 2013; Tang et al., 2014), Rf5 and Rf6 for HL-CMS (Hu et al., 2012b; Huang et al., 2015) have been cloned or finely mapped, and can be well used for molecular breeding. This 
will provide us the possibility to breed wide-spectrum restorer lines that can be used not only for two-line hybrid rice, but also for HL- and WA-type hybrid rice by pyramiding these four $R f$ genes together.

Apart from the strong restoring ability, an elite restorer line should also have high combining ability, large panicles, and strong resistance to diseases and insect pests. Additive effect is one of the important factors that contribute to heterosis of rice yield by the accumulation of genetic effects from multiple dominant or semi-dominant loci (Luo et al., 2009; Birchler, 2015; Dan et al., 2015). Of which, many of rice yield-related genes/QTLs should play a key role on the yield heterosis just as OsPPR37 and Ghd7 do in rice (Liu et al., 2015). Interestingly, Gn8.1, a newly identified gene from wild rice Oryza longistaminata was suggested conferring rice big panicle and high grain yield but little side effects on the rice development, and showed a semi-dominant inheritable model, expression of Gn8.1 can greatly increase grain number and yield (unpublished data). Thus, Gn8.1 has great potential in developing elite restorer lines because of its high yield potential and vast pollen for production of more $\mathrm{F}_{1}$ seeds. Brown planthopper (BPH) is one of the severe destructive insect pests in rice production (Zhao et al., 2016). The utilization of BPH-resistant varieties has been recognized as the most effective and environment-friendly measure against detriment from brown planthopper to rice production (Suh et al., 2011). Today, there are over 30 BPH-resistant genes having been genetically defined (Zhao et al., 2016). Of which, both Bph6 (Qiu et al., 2010) and Bph9 (Zhao et al., 2016), identified from cultivar Swarnalata and Pokkali respectively, are suggested conferring strong resistance to the brown planthopper, and show tremendous potentiality in developing brown planthopperresistant rice varieties.

In order to develop an elite BPH-resistant restorer line with wide restoring ability and good combining ability, we pyramided big-panicle gene Gn8.1, BPH-resistant genes Bph6 and Bph9, fertility restorer genes $R f 3, R f 4, R f 5$, and $R f 6$ into elite rice 9,311 based on molecular marker assisted selection, and successfully developed three elite BPH-resistant wide-spectrum restorer lines. These new restorer lines not only significantly improved the resistance against brown planthopper, but also showed good performance for the production of two-line, HL- and WA-type three line hybrid rice.

\section{MATERIALS AND METHODS}

\section{Plant Materials and Brown Planthopper Population}

9,311, a BPH-susceptible elite restorer line for HL-CMS and two-line hybrids, was used as the recurrent parent. L1880, a chromosome segment substitution line (CSSL) carrying Gn8.1 gene in 9,311 genetic background, was used as the donor parent for large panicle. Shuhui527, an elite restorer line for WA-CMS and two-line hybrids, was used as the donor parent for Wild-Abortive type restorer gene. Luoyang-6 and Luoyang-9, CSSLs carrying Bph6 and Bph9 in 9,311 genetic background, respectively, were used as the donor parents for
BPH-resistance. In addition, seven different types of CMS or GMS sterile lines were used as test parents in this study (Table S1). The brown planthopper populations used for infestation were collected from rice experimental field in Wuhan University.

\section{Evaluation for Plant Resistance against Brown Planthopper}

A seedling bulk test was conducted to evaluate the brown planthopper resistance of rice populations following previously described methods (Huang et al., 2001). The seeds of each rice were pre-germinated to ensure that all seedlings were grown consistently. Detailedly, about 20 seeds were sown in a $9 \mathrm{~cm}$ diameter plastic cup with three replications. At the third-leaf stage, the seedlings were thinned to 10 plants per cup and infested with second to third-instar nymphs of brown planthopper with eight insects per seedling. When all of the seedlings of susceptible control died, the plants of other rice were examined and each seedling was given a score of 1 to 9 according to the method of Huang et al. (2001).

\section{Foreground Selection and Background Profiling by SSR Marker Analysis}

Rice genomic DNA for PCR analysis was extracted from young leaves using the CTAB method (Murray and Thompson, 1980). Seven gene-specific PCR markers, Indel33, RM16994, RM28438, RM10318, RM6100, RM25661, and Indel200-1, tightly linked to the target genes $G n 8.1, B p h 6, B p h 9, R f 3, R f 4, R f 5$, and $R f 6$, respectively, were used in foreground selection of the target genes in each generation of intercross and backcross (Table S2). PCR was performed in a total volume of $10 \mu \mathrm{l}$ containing $50 \mathrm{ng}$ of DNA template, $1 \mu \mathrm{l} 10 \times$ PCR buffer, $0.25 \mu \mathrm{M}$ of each primer, $75 \mu \mathrm{M}$ of each dNTP, and 0.25 units of Taq DNA polymerase (Fermentas). The PCR amplification condition was as follows: one cycle at $94^{\circ} \mathrm{C}$ for $5 \mathrm{~min}$, followed by 36 cycles of $94^{\circ} \mathrm{C}$ for $30 \mathrm{~s}, 50 \sim 60^{\circ} \mathrm{C}$ (dependent on the primers) for $30 \mathrm{~s}, 72^{\circ} \mathrm{C}$ for 45 $\mathrm{s}$, and a final incubation at $72^{\circ} \mathrm{C}$ for $5 \mathrm{~min}$. All the PCR products were analyzed by electrophoresis on agarose gel.

A total of 672 SSR markers distributed evenly on the 12 chromosomes with an average marker interval of $553.4 \mathrm{~kb}$ were used for rice genetic polymorphism survey, and 225 SSR markers were found to be polymorphic between the recurrent and donor parents (Table S3). Then, the 225 markers were used to identify the background of the pyramided lines. The physical map was drawn using MapMaker Version 3.0 (Lander et al., 1987). The percentages of chromosome segments from recurrent parent in pyramided lines were reckoned following the graphical genotyping procedure (Xi et al., 2006; Suh et al., 2013).

\section{Evaluation of Agronomic Traits in the Field}

All of the rice lines were planted in the experimental field of Ezhou in summer, and Hainan in winter during 2011-2016. Among them, the hybrid combinations and their parents used for evaluating the restoring ability and production potential of the new restorer line were planted in the Wuhan University Ezhou Experimental Base in the summer of 2016. Seedlings were transplanted in a five-row plot with 12 plants per row by $20 \times$ 
$26 \mathrm{~cm}$ spacing under a randomized complete block design with three replications. Five representative plants in the middle of each repeat were used to measure growth duration (GD), plant height $(\mathrm{PH})$, panicle number $(\mathrm{PN})$, number of grain $(\mathrm{NG})$, number of grain per panicle (NGP), spikelet fertility (SF), 1,000-grain weight $(\mathrm{GW})$, and yield per plant (YP). The statistical analyses were performed with SPSS Statistics 20 (IBM, USA).

\section{RESULTS}

\section{Development of BPH-Resistant Wide-Spectrum Restorer Lines by Pyramiding}

As shown in the schematic flow (Figure 1) of the development of the BPH-resistant wide-spectrum restorer lines, five parents including L1880, Luoyang-6, Luoyang-9, and typical restorer line Shuhui527 and 9,311 were used in this breeding program. Of which, L1880 derived from crosses of 9,311 $\times$ O. longistaminata carries big panicle gene Gn8.1, Luoyang-6 and Luoyang-9 carry Bph6 and Bph9, respectively, Shuhui527 carries restoring gene $R f 3$ and $R f 4$, and 9,311 carries restoring gene $R f 5$ and $R f 6$. During the breeding process, foreground selection was performed from generation $\mathrm{F}_{1}$ to $\mathrm{BC}_{3} \mathrm{~F}_{3}$ to screen the plants having the desirable alleles of the seven target genes using molecular markers (Table S2). Only progenies carrying all of target alleles were selected for backcross or selfing (Figures S1-S3). Meanwhile, phenotype identification was also conducted so as to eliminate plants with linkage drag traits (Figures S4, S5; Tables S4, S5). In detail, 20 positive $F_{1}$ plants with seven target genes were identified and genotyped using the target gene markers from a $\sim 1,000 \mathrm{~F}_{1}$ population derived from intercross of L1880/Luoyang9//Shuhui527/Luoyang-6. From which, 16 plants were identified having all of the seven target genes (Gn8.1/Bph6/9/Rf3 6), and further backcrossed as maternal parents with 9,311. In the $\mathrm{BC}_{1}$ generation, 18 out of 890 plants were identified as our expected candidates after careful genotyping and phenotyping. Similarly, the selected $\mathrm{BC}_{1}, \mathrm{BC}_{2}$ plants were then backcrossed with 9,311 so as to eliminate the unexpected background. Then, $29 \mathrm{BC}_{3} \mathrm{~F}_{1}$ plants were selected for selfing until genetically stable in $\mathrm{BC}_{3} \mathrm{~F}_{3}$. Finally, 22 stable $\mathrm{BC}_{3} \mathrm{~F}_{3}$ lines with target genes were developed from 13,580 plants. In consideration of the integrative agronomic traits and the presence of homozygous marker alleles for the seven target genes, 16 lines were selected as potential candidates from the $22 \mathrm{BC}_{3} \mathrm{~F}_{3}$ progenies (Figure S3; Table S5).

\section{Evaluation of Brown \\ Planthopper-Resistance and Agronomic Traits of Pyramided Lines}

To test whether the selected pyramiding lines can improve the $\mathrm{BPH}$-resistance, we evaluated their resistance to brown planthopper at seedling stage under greenhouse conditions, using Luoyang-6 and Luoyang-9 (donor parents) as resistant control, and 9,311 (recurrent parent) as a susceptible control. After inoculated for 1 week, when all the 9,311 seedlings were dead, the pyramided lines were still green (Figure 2A). Resistance scoring showed that $\mathrm{BPH}$-resistance indexes of the part of pyramided lines ranged from 1.0 to 1.8 , significantly less than that of the Luoyang-6 (3.2) and Luoyang-9 (3.3) (Figure 2B). This result implied that these pyramided lines' $\mathrm{BPH}$-resistance were significantly stronger than that of the single-gene donor parents Luoyang- 6 and Luoyang-9, although both of them showed high resistant level.

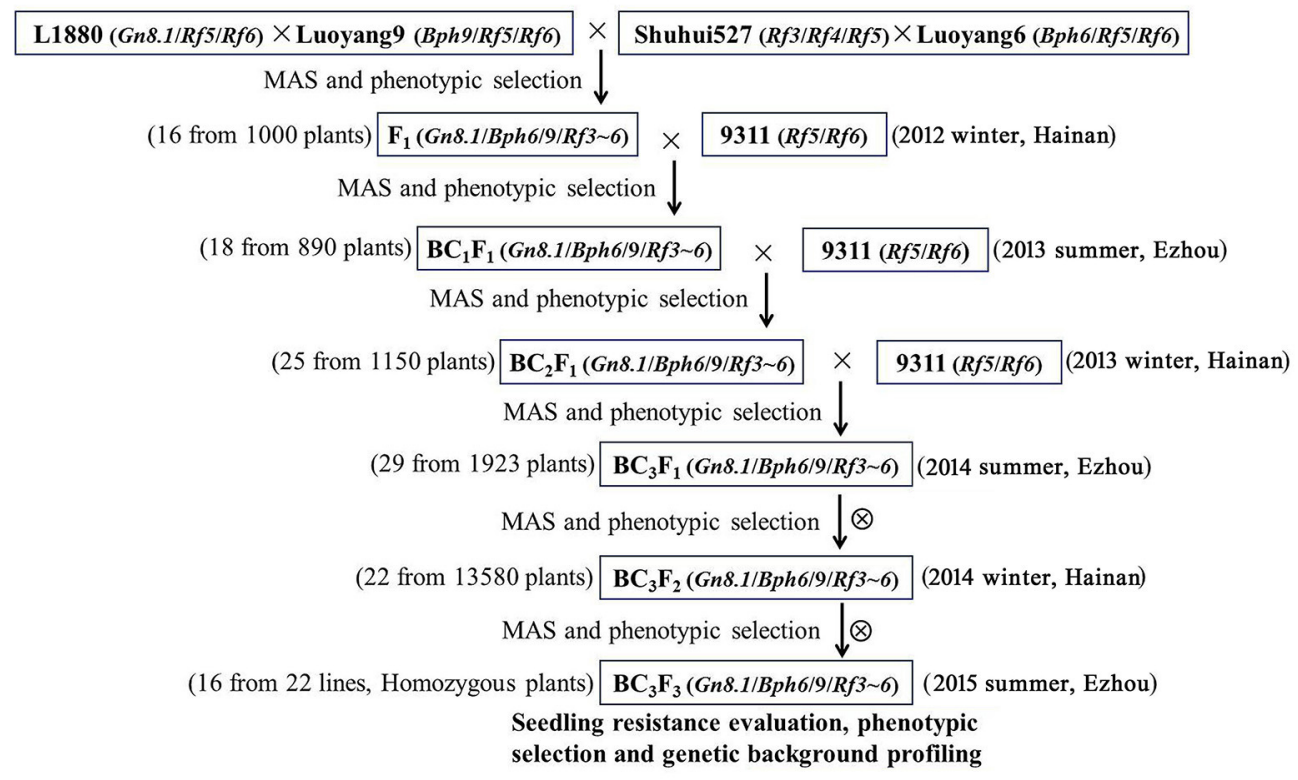

FIGURE 1 | Schematic flow for the development of BPH-resistant wide-spectrum restorer lines. 
Importantly, comparing with the recurrent parent 9,311, these pyramided lines, especially line 05,06 , and 08 all showed excellent performance in the field test. The traits such as growth period, plant height, panicle number and 1,000-grain weight of the three pyramided lines were almost the same as that of 9,311. However, the panicle size and grain number of 05 , 06 , and 08 were significantly larger than that of 9,311. This is a desirable agronomic trait for increasing yield. As a result, even if the spikelet fertility of 05,06 , and 08 were slightly declined, their yield per plant were still increased by 77.3 , 66.1 , and $85.1 \%$, respectively than that of 9,311 (Table 1). Thus, the pyramided lines 05,06 , and 08 were finally chosen as the potential elite BPH-resistant restorer lines based on their excellent performance, and renamed as R345, R346, and R348, respectively (Figure 3 ).

\section{Genetic Background Profiling of the Pyramided Lines}

In order to identify the genetic background of the three pyramided lines, a total of 672 evenly distributed SSR markers were used for PCR identification, and physical maps covering the whole 12 rice chromosomes were constructed (Figure 4). The results showed that each pyramided line carried part of the donors' fragments at the seven target gene loci, even after three rounds of backcrossing with the recurrent parent 9,311. Averagely, the 9,311 genomic fragments took over 91.4, 87.5, and $88.3 \%$ in pyramided lines R345, R346, and R348, respectively (Table S3). These results demonstrated that the seven target genes Gn8.1, Bph6, Bph9, Rf3, Rf4, Rf5, and Rf6 was successfully pyramided together through molecular marker selection, and R345 had the cleanest genetic background.
A

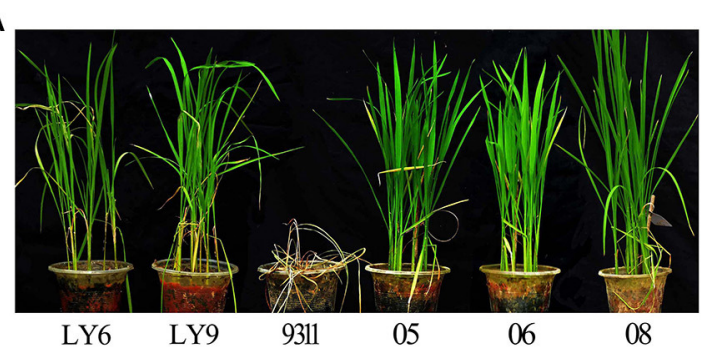

C

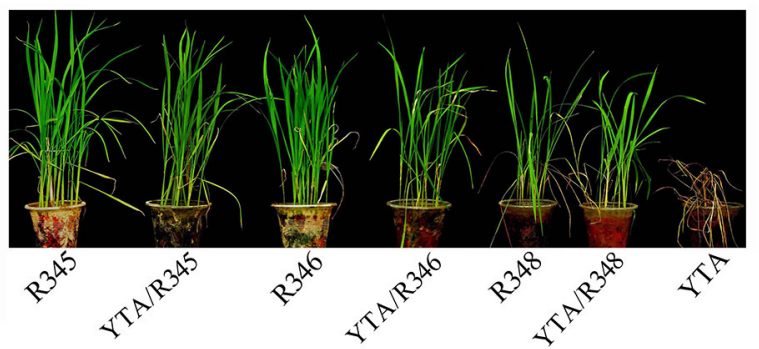

B

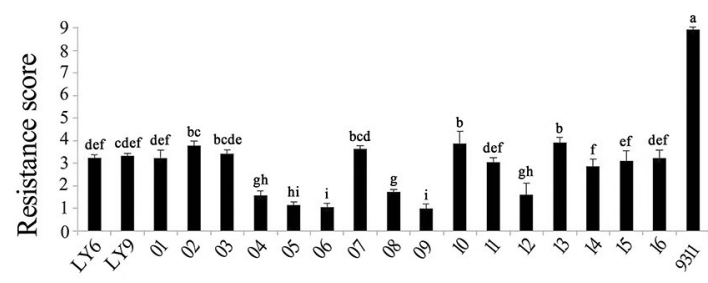

D

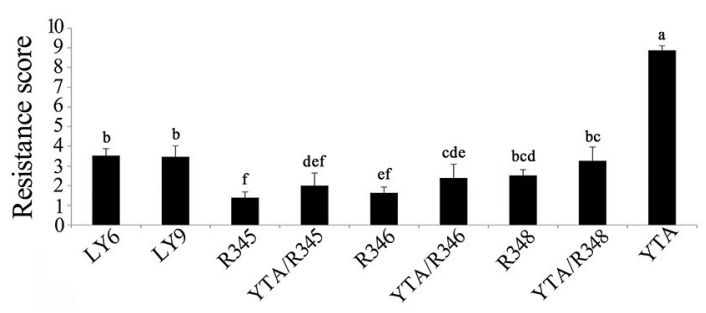

FIGURE 2 | BPH-resistance test of the BPH-resistant wide-spectrum restorer lines at the seedling stage. (A,B): BPH-resistance phenotype (A) and scores (B) of the pyramided restorer lines. LY6 and LY9, Resistant control line Luoyang-6 and Luoyang-9, respectively (The same as below); 9,311, susceptible control; 01 16: BC 3 F 3 lines. (C,D): BPH-resistance test (C) and scores (D) of the restorer lines and hybrid rice $F_{1}$ s. YTA, Susceptible control Yuetai A. Letters indicate a significant difference at the $5 \%$ significance level by the least significant difference test.

TABLE 1 | Agronomic traits of the BPH-resistant wide-spectrum restorer lines and parents.

\begin{tabular}{|c|c|c|c|c|c|c|c|c|}
\hline Rice lines & GD & PH (cm) & PN & NG & NGP & SF (\%) & GW (g) & YP (g) \\
\hline 9311 & $130 \pm 1.0^{b}$ & $123 \pm 6^{\mathrm{bc}}$ & $7.0 \pm 1.0^{\mathrm{C}}$ & $1193 \pm 133^{C}$ & $171 \pm 6^{b}$ & $92.2 \pm 0.8^{a}$ & $29.2 \pm 0.2^{a}$ & $32.2 \pm 4.0^{C}$ \\
\hline L1880 & $132 \pm 1.0^{\mathrm{a}}$ & $126 \pm 5^{b}$ & $7.0 \pm 1.0^{\mathrm{C}}$ & $2018 \pm 333^{b}$ & $288 \pm 8^{a}$ & $88.6 \pm 1.3^{b}$ & $29.4 \pm 0.4^{a}$ & $52.3 \pm 7.4^{b}$ \\
\hline SH527 & $122 \pm 0.6^{d}$ & $120 \pm 4^{c}$ & $8.2 \pm 0.3^{a}$ & $1352 \pm 28^{C}$ & $169 \pm 3^{b}$ & $87.3 \pm 1.8^{\mathrm{bcd}}$ & $29.3 \pm 0.4^{a}$ & $34.5 \pm 0.6^{c}$ \\
\hline LY6 & $132 \pm 0.6^{a}$ & $130 \pm 7^{a}$ & $7.0 \pm 1.0^{\mathrm{C}}$ & $1215 \pm 121^{\mathrm{C}}$ & $174 \pm 8^{b}$ & $85.8 \pm 2.9^{d}$ & $29.7 \pm 0.1^{a}$ & $31.1 \pm 4.2^{\mathrm{C}}$ \\
\hline LY9 & $130 \pm 0.6^{b}$ & $131 \pm 5^{a}$ & $7.7 \pm 0.6^{\mathrm{abc}}$ & $1305 \pm 102^{C}$ & $170 \pm 1^{b}$ & $86.4 \pm 0.7^{\mathrm{cd}}$ & $29.8 \pm 0.9^{a}$ & $33.6 \pm 3.7^{c}$ \\
\hline R345 & $130 \pm 1.0^{b}$ & $127 \pm 8^{a b}$ & $7.3 \pm 0.6^{b c}$ & $2180 \pm 249^{a b}$ & $297 \pm 12^{a}$ & $88.3 \pm 1.1^{b c}$ & $29.7 \pm 0.7^{a}$ & $57.1 \pm 5.9^{a b}$ \\
\hline R346 & $128 \pm 1.0^{c}$ & $126 \pm 2^{b}$ & $7.0 \pm 0.8^{c}$ & $2028 \pm 101^{b}$ & $290 \pm 14^{a}$ & $89.1 \pm 1.3^{b}$ & $29.6 \pm 1.1^{a}$ & $53.5 \pm 1.2^{b}$ \\
\hline R348 & $130 \pm 0.6^{b}$ & $123 \pm 7^{\mathrm{bc}}$ & $8.1 \pm 0.2^{\mathrm{ab}}$ & $2320 \pm 121^{a}$ & $290 \pm 15^{a}$ & $88.4 \pm 2.7^{b c}$ & $29.1 \pm 0.7^{a}$ & $59.7 \pm 1.5^{\mathrm{a}}$ \\
\hline
\end{tabular}

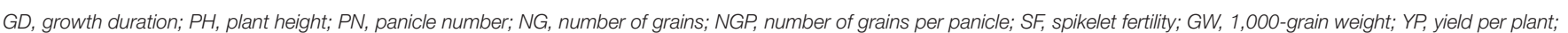

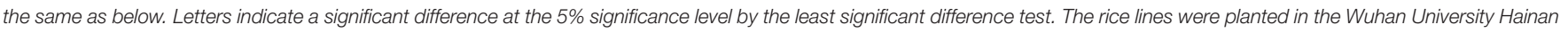
Experimental Base in the winter of 2015. 

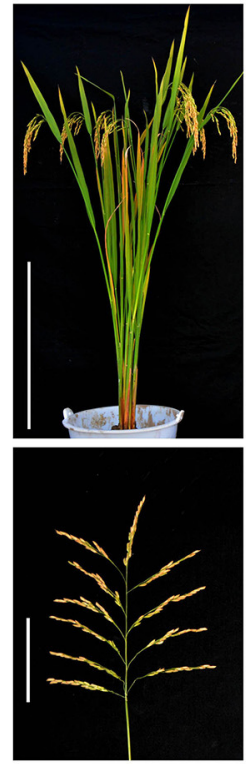

9311
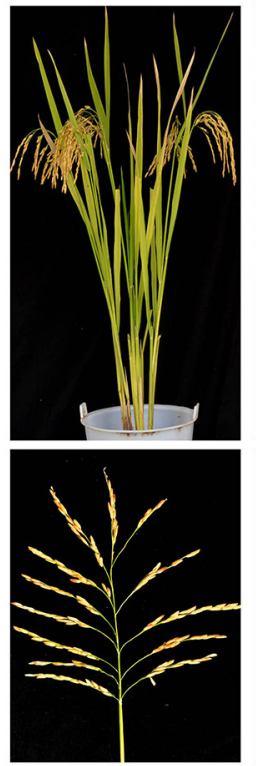

SH527
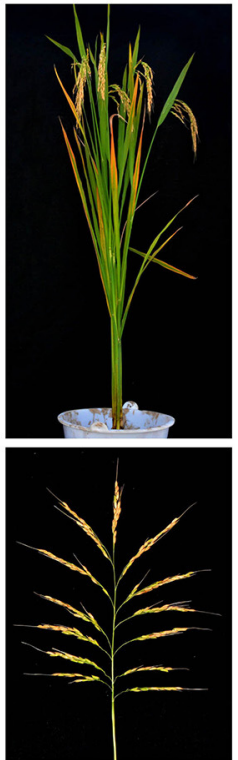

LY6
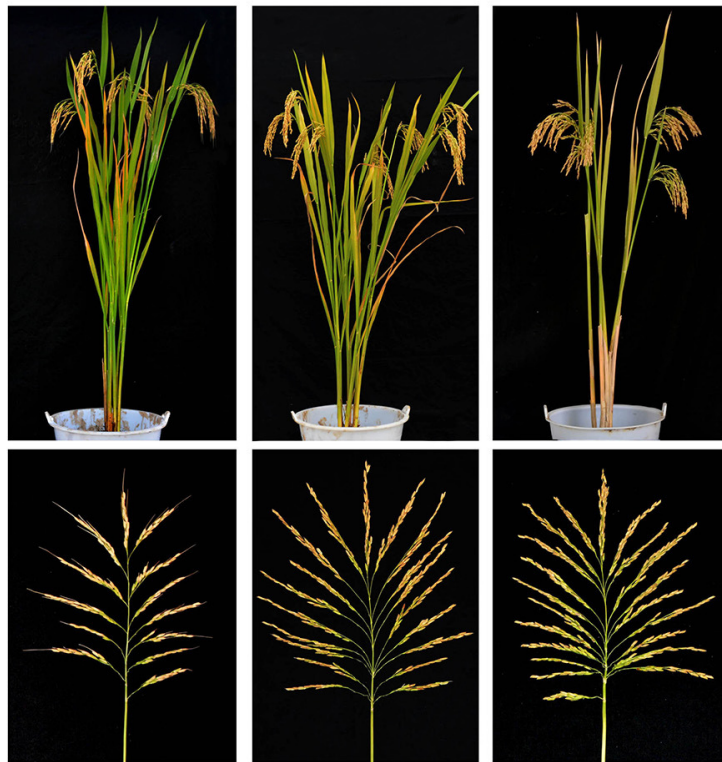

LY9

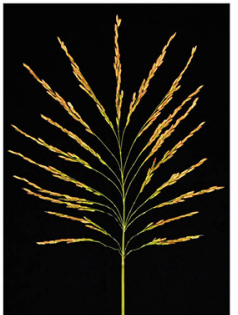

R345

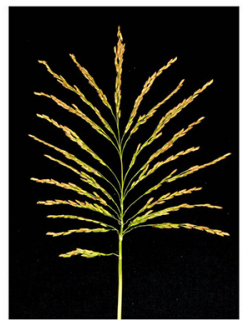

L1880

FIGURE 3 | Gross plant morphology of the parent lines and newly developed BPH-resistant wide-spectrum restorer line R345. Upper panel represents plant stature, scale bar $=50 \mathrm{~cm}$; lower panel represents panicle, scale bar $=10 \mathrm{~cm}$.
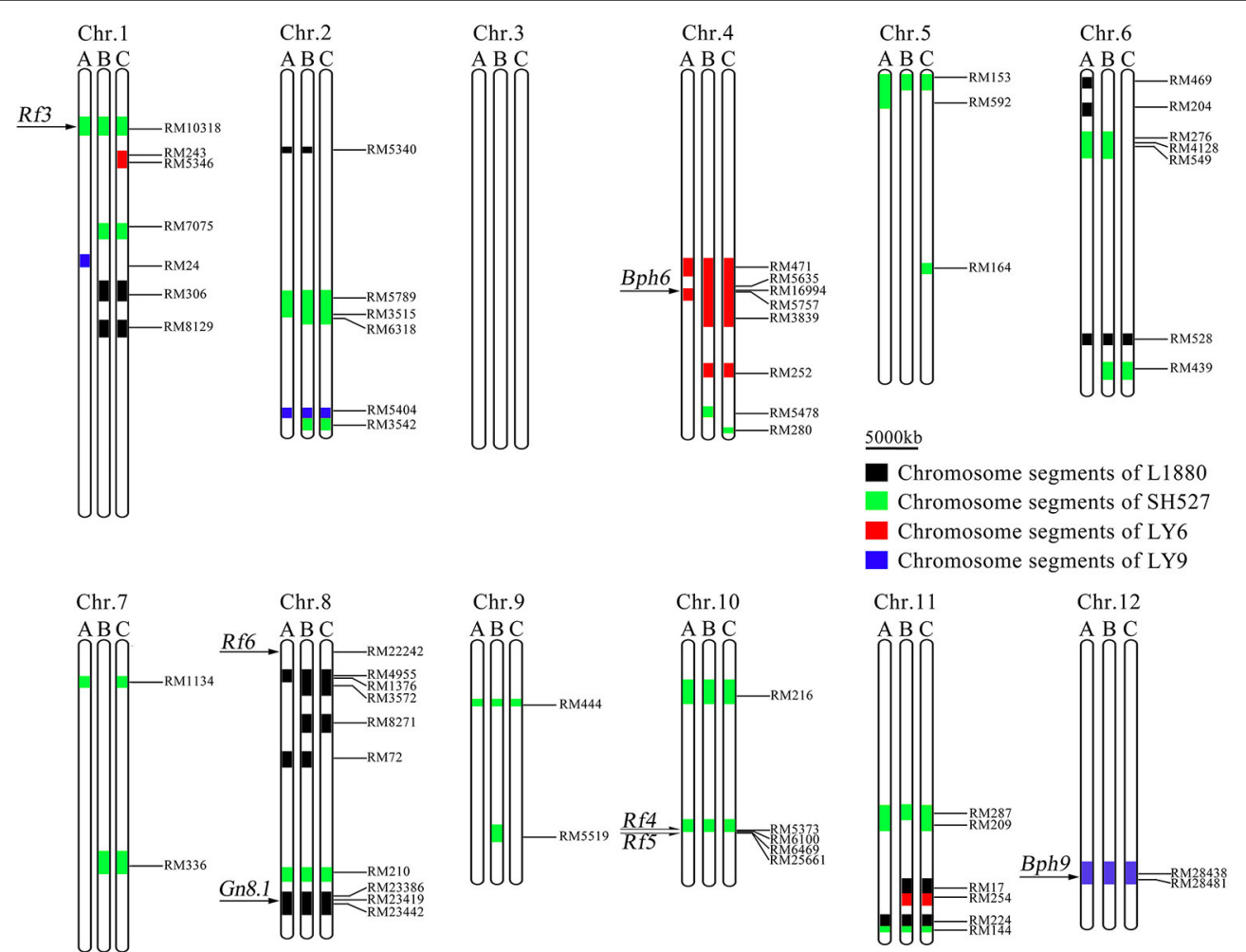

FIGURE 4 | Genotypes of three newly developed BPH-resistant wide-spectrum restorer lines under 9,311 genetic background. Letters A, B and C represent line R345, R346, and R348, respectively. The black, green, red, and blue boxes indicate substituted chromosome segments of the donor parents L1880, Shuhui527, Luoyang-6, and Luoyang-9, respectively. 


\section{Evaluation of Fertility Restoring Ability of Pyramided Lines}

In order to evaluate the restoring ability of the three pyramided lines, we examined the pollen and spikelet fertility of the testcrosses derived from the pyramided restorer lines. All of the six HL-type hybrid crosses (HL-CMS lines Luohong-4A and Jiahong-2A crossed with R345, R346, and R348, respectively) showed the same pollen fertility phenotype as the typical HL-type hybrid combinations Luohong-4A/9311 and Jiahong-2A/9311. Similarly, all of the WA-type and two-line hybrid combinations showed the same pollen fertility as their respective controls, and reached to almost 100\% (Figure 5A; Figure S6). Moreover, we observed that the spikelet fertility showed a similar trend as that of the pollen fertility (Figure 5B; Figure S7). These results showed that the pyramided lines R345, R346, and R348 could restore all types of sterile lines, implying these elite BPH-resistant lines had wide-spectrum restoring ability.

\section{Performance of the Testcrosses Derived from the New Restorer Lines}

To determine if the newly developed BPH-resistant widespectrum restorer lines can be well used in hybrid rice production, 30 hybrid rice crosses were constructed by using R345, R346, and R348, and parent restorers 9,311 (for HLCMS and two-line) and SH527 (for WA-CMS and two-line) to testcross HL-CMS lines Luohong-4A and Jiahong-2A, WACMS lines Yuexiang-A and Luofei- $\mathrm{A}$, and two-line sterile lines BPH68S and Guangzhan4S, respectively. Rice yields and agronomic traits including plant height, panicle number, number of grains per panicle, spikelet fertility, and 1,000-grain weight were examined when matured (Table 2; Table S6). Relative to the control of HL-type hybrid rice Luohong-4A/9311, number of grains, number of grains per panicle, and yield per plant of new HL-type combinations Luohong-4A/R345, Luohong-4A/R346, and Luohong-4A/R348 significantly increased. Similarly, for the WA-CMS, hybrid crosses derived from Yuexiang-A and restorer lines R345, R346, and R348 performed better than that of the testcrosses derived from restorer line SH527, showing significant improvements in yield per plant, although the growth duration of the combinations from Yuexiang-A crossed with the three new restorer lines were prolonged. For the two-line hybrid rice, the crosses derived from the three new restorer lines also showed significantly yield advantage over the controls. Then, we tested the resistance of the hybrid rice derived from R345, R346, and R348 and HL-CMS line Yuetai-A (BPHsusceptible), respectively. When the Yuetai-A seedlings almost completely died, Yuetai-A/R345, Yuetai-A/R346, and YuetaiA/R348 showed normal or only slightly endangered symptoms (Figure 2C), and even a higher resistant level than the resistant controls (Figure 2D). These results indicated that the hybrid rice derived from the new restorer lines not only had strong resistance to the brown planthopper, but also high yield potential.

\section{DISCUSSION}

Sustainable development of hybrid rice depended greatly on the construction of elite CMS/GMS lines and restorers. Pyramiding multiple favorable traits in a commercial restorer is considered to be one of the most effective approaches to create elite restorer lines. However, it is often difficult to reach such a goal using traditional breeding methods because of the inefficient selection and unexpected linkage drags. Thus, the molecular assisted selection becomes an important strategy in rice breeding programs for the advantage of rapid recovery of the background of the recurrent parents (Suh et al., 2013; Fan et al., 2015;

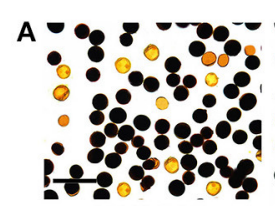

LH4A/9311

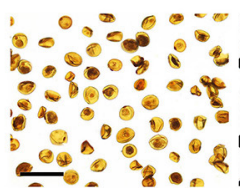

YXA/9311

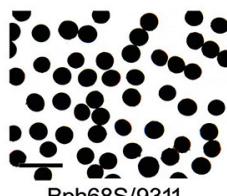

Bph68S/9311

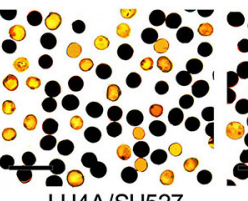

LH4A/SH527

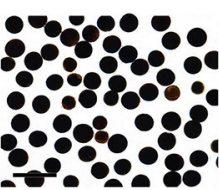

YXA/SH527

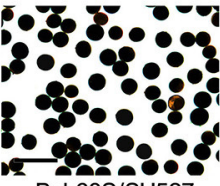

Bph68S/SH527

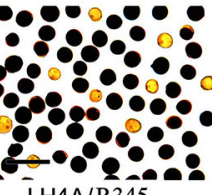

LH4A/R345

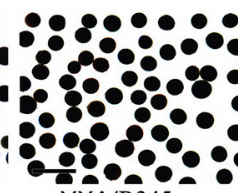
YXA/R345

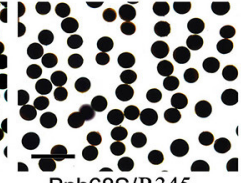

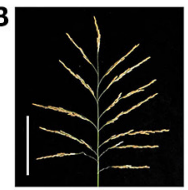

LH4A/9311

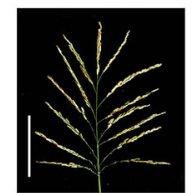

YXA/9311

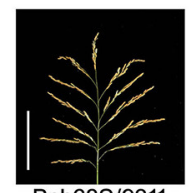

Bph68S/9311

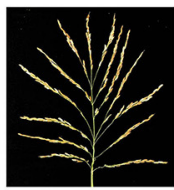

LH4A/SH527

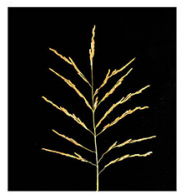

YXA/SH527

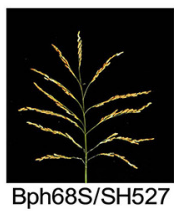

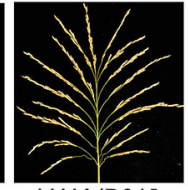

LH4A/R345
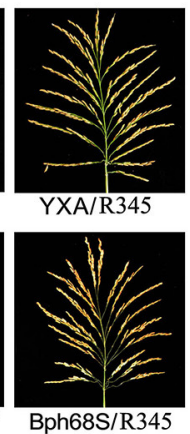

FIGURE 5 | Restoring ability of the newly developed BPH-resistant wide-spectrum restorer lines in HL-CMS, WA-CMS, and two-line male sterile lines. (A) Pollen fertility of $F_{1} s$ assessed by $1 \% I_{2}-\mathrm{KI}$ staining. Darkly stained pollen is fertile, and slightly stained pollen is sterile, scale bar $=50 \mu \mathrm{m}$. LH4A, YXA and BPH68S were representative HL-CMS, WA-CMS, and two-line sterile rice, respectively. 9,311: a representative restorer line for HL-CMS and two-line GMS rice, SH527: a representative restorer line for WA-CMS and two-line GMS rice. (B) Mature panicles of hybrid rice $\mathrm{F}_{1} \mathrm{~s}, \mathrm{scale}$ bar $=10 \mathrm{~cm}$. 
TABLE 2 | Agronomic traits of the hybrid rice combinations derived from BPH-resistant wide-spectrum restorer lines and commercial sterile lines.

\begin{tabular}{|c|c|c|c|c|c|c|c|c|}
\hline Combinations & GD & $\mathrm{PH}(\mathrm{cm})$ & PN & NG & NGP & SF (\%) & GW (g) & YP (g) \\
\hline LH4A/9311 & $128 \pm 1.0^{\mathrm{cd}}$ & $125 \pm 4^{\mathrm{bc}}$ & $7.3 \pm 0.6^{\mathrm{cd}}$ & $1543 \pm 74^{g h i}$ & $211 \pm 14^{\text {de }}$ & $74.6 \pm 2.5^{d}$ & $25.7 \pm 0.9^{b c d e}$ & $29.6 \pm 1.0^{f g}$ \\
\hline LH4A/SH527 & $127 \pm 2.0^{d}$ & $120 \pm 4^{\text {de }}$ & $9.7 \pm 0.6^{a}$ & $2120 \pm 258^{\text {bcde }}$ & $219 \pm 18^{d}$ & $49.2 \pm 3.6^{\mathrm{e}}$ & $24.7 \pm 0.5^{f g}$ & $25.6 \pm 1.09$ \\
\hline LH4A/R345 & $128 \pm 0.6^{c d}$ & $124 \pm 2^{\mathrm{cd}}$ & $9.3 \pm 0.6^{a b}$ & $2529 \pm 31^{a}$ & $271 \pm 14^{a b c}$ & $76.2 \pm 2.0^{\mathrm{cd}}$ & $25.6 \pm 0.6^{\text {def }}$ & $49.3 \pm 1.1^{\mathrm{ab}}$ \\
\hline LH4A/R346 & $128 \pm 0.6^{\mathrm{cd}}$ & $123 \pm 2^{\mathrm{cd}}$ & $8.0 \pm 1.0^{\mathrm{abcd}}$ & $2081 \pm 203^{\text {cde }}$ & $261 \pm 14^{\mathrm{bc}}$ & $77.6 \pm 2.7^{\mathrm{bcd}}$ & $25.1 \pm 0.6^{e f g}$ & $40.4 \pm 1.8^{c d}$ \\
\hline LH4A/R348 & $127 \pm 1.0^{d}$ & $125 \pm 2^{\mathrm{C}}$ & $9.3 \pm 1.2^{\mathrm{ab}}$ & $2518 \pm 321^{a b}$ & $270 \pm 15^{a b c}$ & $76.6 \pm 5.1^{\mathrm{bcd}}$ & $25.3 \pm 0.7^{\mathrm{ef}}$ & $48.4 \pm 1.9^{a b}$ \\
\hline YXA/9311 & $121 \pm 1.2^{f}$ & $120 \pm 2^{\text {de }}$ & $7.7 \pm 0.6^{b c d}$ & $1929 \pm 112^{\text {defg }}$ & $252 \pm 10^{c}$ & $0^{f}$ & - & - \\
\hline YXA/SH527 & $114 \pm 0.6^{9}$ & $117 \pm 3^{\mathrm{e}}$ & $8.7 \pm 1.5^{\mathrm{abcd}}$ & $1639 \pm 215^{f g h i}$ & $190 \pm 9^{e f}$ & $81.0 \pm 3.3^{a b}$ & $25.7 \pm 0.4^{\text {bcde }}$ & $34.0 \pm 3.0^{e f}$ \\
\hline YXA/R345 & $123 \pm 0.6^{e}$ & $117 \pm 2^{\mathrm{e}}$ & $8.0 \pm 1.0^{\mathrm{abcd}}$ & $2295 \pm 153^{a b c d}$ & $288 \pm 19^{a}$ & $83.6 \pm 3.7^{\mathrm{a}}$ & $27.0 \pm 0.5^{a}$ & $51.7 \pm 0.6^{a}$ \\
\hline YXA/R346 & $122 \pm 1.0^{\mathrm{ef}}$ & $117 \pm 2^{\mathrm{e}}$ & $7.7 \pm 1.2^{\mathrm{bcd}}$ & $2129 \pm 290^{\text {abcde }}$ & $278 \pm 4^{a b}$ & $80.7 \pm 3.1^{a b c}$ & $25.6 \pm 0.2^{\text {cde }}$ & $43.9 \pm 4.5^{b c}$ \\
\hline YXA/R348 & $121 \pm 1.2^{f}$ & $116 \pm 4^{e}$ & $7.3 \pm 0.6^{\mathrm{cd}}$ & $1999 \pm 136^{\text {def }}$ & $273 \pm 19^{a b c}$ & $82.9 \pm 1.6^{a}$ & $24.2 \pm 0.69$ & $40.1 \pm 2.6^{\mathrm{cd}}$ \\
\hline Bph68S/9311 & $136 \pm 0.7^{a}$ & $131 \pm 2^{\mathrm{a}}$ & $7.7 \pm 1.5^{\mathrm{bcd}}$ & $1385 \pm 131^{i}$ & $183 \pm 21^{f}$ & $77.3 \pm 1.9^{\mathrm{bcd}}$ & $26.8 \pm 0.6^{a}$ & $28.7 \pm 3.1^{\mathrm{fg}}$ \\
\hline Bph68S/SH527 & $129 \pm 1.5^{\mathrm{bc}}$ & $129 \pm 5^{a b}$ & $8.7 \pm 1.2^{\mathrm{abcd}}$ & $1510 \pm 145^{h i}$ & $175 \pm 12^{f}$ & $75.2 \pm 2.9^{d}$ & $26.5 \pm 0.4^{\mathrm{abc}}$ & $30.1 \pm 2.7^{f g}$ \\
\hline Bph68S/R345 & $131 \pm 1.2^{b}$ & $132 \pm 3^{a}$ & $9.0 \pm 1.7^{\mathrm{abc}}$ & $2462 \pm 555^{a b c}$ & $272 \pm 11^{a b c}$ & $73.9 \pm 2.8^{d}$ & $26.5 \pm 0.6^{a b c d}$ & $47.9 \pm 9.5^{a b}$ \\
\hline Bph68S/R346 & $128 \pm 0.6^{c d}$ & $131 \pm 3^{a}$ & $7.3 \pm 0.6^{\mathrm{cd}}$ & $1851 \pm 216^{\text {efgh }}$ & $252 \pm 11^{\mathrm{C}}$ & $74.7 \pm 1.7^{d}$ & $26.7 \pm 0.5^{a}$ & $36.8 \pm 3.1^{\mathrm{de}}$ \\
\hline Bph68S/R348 & $123 \pm 1.5^{\mathrm{e}}$ & $132 \pm 3^{a}$ & $7.0 \pm 1.7^{\mathrm{d}}$ & $1830 \pm 270^{\text {efgh }}$ & $265 \pm 26^{a b c}$ & $76.2 \pm 3.0^{\mathrm{bcd}}$ & $26.5 \pm 0.3^{a b}$ & $36.8 \pm 3.6^{\mathrm{de}}$ \\
\hline
\end{tabular}

GD, growth duration; PH, plant height; PN, panicle number; NG, number of grains; NGP, number of grains per panicle; SF, spikelet fertility; GW, 1000-grain weight; YP, yield per plant.

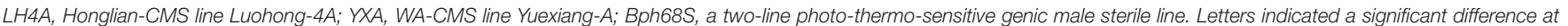

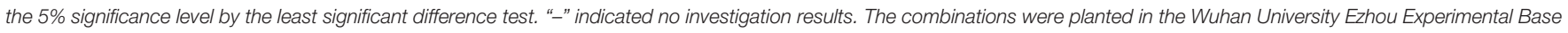
in the summer of 2016 .

Shamsudin et al., 2016). In this study, seven favorable genes were pyramided into 9,311 by using tightly linked molecular markers within six generations. For the reason, apart from the use of CSSLs with the genetic background of recurrent parent 9,311 which can accelerate to purify the genetic background, phenotypic selection that is combined with genotyping with the molecular markers that are tightly linked to the target genes can quickly reduce the linkage drag which may show unexpected side effects on the plant performance. Thus, the newly developed restorer lines showed good performance in the two-line and three-line hybrid rice production for their desirable characters including BPH-resistance, wide restoring ability, and high yield potentiality.

It is well-known that HL- and WA-CMS can be restored by $R f 3 / R f 4$ and $R f 5 / R f 6$ gene pairs, respectively (Luo et al., 2013; Huang et al., 2015). Different fertility restoring patterns limit exploitation of the heterosis potential of the three-line hybrid rice. If pyramiding of these $R f$ genes together to construct a widespectrum restorer line will break the isolation between different CMS types. Although gene pyramiding has been successfully used to improve rice grain yield, quality, and resistance in the last decades (Hittalmani et al., 2000; Zhou et al., 2003; Kumar et al., 2016), no reports are openly available for breeding widespectrum restorers by pyramiding of multiple $R f$ genes. In this study, the restorer lines carrying $R f 3, R f 4, R f 5$, and $R f 6$ showed good fertility restoration for WA- and HL- three-line hybrid rice, and also two-line hybrid rice (Figure 5). It implies that these four $R f$ genes are functionally compatible each other, and these wide-spectrum restorers can be effectively used for breeding of different type hybrid rice.

Apart from high fertility restoring ability, strong resistance of the parents to diseases and insect pests are also important requirements for sustainable development of hybrid rice. As for brown planthopper, it is well recognized that the most efficient way to conquer this pest is to breed $\mathrm{BPH}$-resistant rice by pyramiding multiple functional genes against brown planthopper. It is reported that rice carried Bph3 and Bph27(t) had higher resistance than $B p h 3$ or $B p h 27(t)$ introgression lines (Liu et al., 2016). Rice pyramided Bph14, Bph15, and Bph18 genes, similarly, showed higher resistance than those two genes pyramided lines, and the two genes pyramided lines also higher than single gene lines ( $\mathrm{Hu}$ et al., 2012a, 2013). In this study, Bph6 and Bph9 were selected as target genes against brown planthopper, due to their advantages in BPH-resistance. Of which, Bph 9 shows durable and broad resistance to brown planthopper biotypes 1, 2, and 3 (Zhao et al., 2016). Bph6 is also a high BPH-resistant gene, especially against brown planthopper biotype 4 (Qiu et al., 2010). As observed in the test, the restorer lines with both Bph6 and Bph9 and even their hybrid rice all showed higher resistance than any of the single-gene donor parents (Figure 2). It means that Bph6 and Bph 9 together can confer rice broad, higher and durable resistance to all four brown planthopper biotypes, and these two genes had great potential in future hybrid rice breeding programs.

\section{CONCLUSIONS}

We developed three elite BPH-resistant wide-spectrum restorer lines by multiple genes pyramiding through MAS. These new restorer lines significantly improved the resistance against $\mathrm{BPH}$, and showed good performance in the two-line and three-line hybrid rice production. Therefore, it can be concluded that the development of elite $\mathrm{BPH}$-resistant wide-spectrum restorer lines had a promising future for breeding of broad spectrum BPHresistant high-yield varieties. 


\section{AUTHOR CONTRIBUTIONS}

SL, YZ, GH, and FF designed the research; FF, NL, YC, XL, HS, and JW performed molecular and field experiments; FF and SL analyzed data and wrote the manuscript. All authors read and approved the final manuscript.

\section{ACKNOWLEDGMENTS}

This research was partly supported by the National Transgenic Research and Development Program (2016ZX08001004-001002), the 863 Program (2014AA10A604-9), Basic Research Program of Wuhan City (2015020101010078), and Natural Science Foundation (31370363) of China.

\section{SUPPLEMENTARY MATERIAL}

The Supplementary Material for this article can be found online at: http://journal.frontiersin.org/article/10.3389/fpls.2017. 00986/full\#supplementary-material

Figure S1 | PCR analysis of the parental lines and $F_{1}$ plants. M, DNA marker DL2000, the same as below. (A) Indel33 for Gn8.1. (B) RM16994 for Bph6. (C) RM28438 for Bph 9. (D) RM10318 for Rf3. (E) RM6100 for Rf4. (F) RM25661 for Rf5. (G) Indel200-1 for Rf6.

\section{REFERENCES}

Birchler, J. A (2015). Heterosis: the genetic basis of hybrid vigour. Nat. Plants 1:15020. doi: 10.1038/nplants.2015.20

Dan, Z., Hu, J., Zhou, W., Yao, G., Zhu, R., Huang, W., et al. (2015). Hierarchical additive effects on heterosis in rice (Oryza sativa L.). Front. Plant Sci. 6:738. doi: $10.3389 /$ fpls. 2015.00738

Fan, F. F., Li, N. W., Wang, J., Liu, X. D., Liu, J. F., Zhu, Y. G., et al. (2015). Molecular marker-directed development of a novel cytoplasmic male sterile line in rice. Mol. Breed. 35:212. doi: 10.1007/s11032-015-0394-2

Hittalmani, S., Parco, A., Mew, T. V., Zeigler, R. S., and Huang, N. (2000). Fine mapping and DNA marker-assisted pyramiding of the three major genes for blast resistance in rice. Theor. Appl. Genet. 100, 1121-1128. doi: 10.1007/s001220051395

Hu, J., Cheng, M., Gao, G., Zhang, Q., Xiao, J., and He, Y. (2013). Pyramiding and evaluation of three dominant brown planthopper resistance genes in the elite indica rice 9311 and its hybrids. Pest. Manag. Sci. 69, 802-808. doi: $10.1002 / \mathrm{ps} .3437$

Hu, J., Li, X., Wu, C. J., Yang, C. J., Hua, H. X., Gao, G. J., et al. (2012a). Pyramiding and evaluation of the brown planthopper resistance genes Bph14 and Bph15 in hybrid rice. Mol. Breed. 29, 61-69. doi: 10.1007/s11032-010-9526-x

Hu, J., Wang, K., Huang, W., Liu, G., Gao, Y., Wang, J., et al. (2012b). The rice pentatricopeptide repeat protein RF5 restores fertility in Hong-Lian cytoplasmic male-sterile lines via a complex with the glycine-rich protein GRP162. Plant Cell 24, 109-122. doi: 10.1105/tpc.111.093211

Huang, W., Yu, C., Hu, J., Wang, L., Dan, Z., Zhou, W., et al. (2015). Pentatricopeptide-repeat family protein RF6 functions with hexokinase 6 to rescue rice cytoplasmic male sterility. Proc. Natl. Acad. Sci. U.S.A. 112, 14984-14989. doi: 10.1073/pnas.1511748112

Huang, Z., He, G., Shu, L., Li, X., and Zhang, Q. (2001). Identification and mapping of two brown planthopper resistance genes in rice. Theor. Appl. Genet. 102, 929-934. doi: 10.1007/s001220000455

Kumar, V. A., Balachiranjeevi, C. H., Naik, S. B., Rambabu, R., Rekha, G., Harika, G., et al. (2016). Development of gene-pyramid lines of the elite restorer line, RPHR-1005 Possessing durable bacterial blight and blast resistance. Front. Plant Sci. 7:1195. doi: 10.3389/fpls.2016.01195
Figure S2 I PCR analysis of the parental lines and $\mathrm{BC}_{3} \mathrm{~F}_{2}$ plants. (A) Indel33 for Gn8. 1. (B) RM16994 for Bph 6. (C) RM28438 for Bph 9. (D) RM10318 for Rf3. (E) RM6100 for Rf4. (F) RM25661 for Rf5. (G) Indel200-1 for Rf6.

Figure S3 | PCR analysis of the parental and $\mathrm{BC}_{3} \mathrm{~F}_{3}$ lines. (A) Indel33 for Gn8.1. (B) RM16994 for Bph6. (C) RM28438 for Bph9. (D) RM10318 for Rf3. (E) RM6100 for Rf4. (F) RM25661 for Rf5. (G) Indel200-1 for Rf6.

Figure S4 | Gross plant (Upper panel) and panicle (Lower panel) morphologies of rice. Scale bars represent 50 and $10 \mathrm{~cm}$, respectively.

Figure S5 | Gross plant (Upper panel) and panicle (Lower panel) morphologies of rice. Scale bars represent 50 and $10 \mathrm{~cm}$, respectively.

Figure S6 | Pollen fertility of hybrid combinations. (A) HL-type hybrid combinations. (B) WA-type hybrid combinations. (C) two-line hybrid combinations.

Figure S7 | Spikelet fertility of hybrid combinations. (A) HL-type hybrid combinations. (B) WA-type hybrid combinations. (C) two-line hybrid combinations.

Table S1 | Rice materials used in this study.

Table S2 | Markers used for foreground selection of seven target genes in this study.

Table S3 | Genotyping of the newly developed BPH-resistant wide-spectrum restorer lines.

Table S4 | Agronomic traits performance of the parental lines and hybrid plants.

Table S5 | Agronomic traits performance of the parents and $\mathrm{BC}_{3} \mathrm{~F}_{3}$ lines.

Table S6 | Agronomic traits of $F_{1} s$.

Lander, E. S., Green, P., Abrahamson, J., Barlow, A., Daly, M. J., Lincoln, S. E., et al. (1987). MAPMAKER: an interactive computer package for constructing primary genetic linkage maps of experimental and natural populations. Genomics 1, 174-181. doi: 10.1016/0888-7543(87)90010-3

Li, S., Yang, D., and Zhu, Y. (2007). Characterization and use of male sterility in hybrid rice breeding. J. Integr. Plant Biol. 49, 791-804. doi: 10.1111/j.1744-7909.2007.00513.x

Li, S., Yang, G., Li, S., Li, Y., Chen, Z., and Zhu, Y. (2005). Distribution of fertility-restorer genes for wild-abortive and Honglian CMS lines of rice in the AA genome species of genus Oryza. Ann. Bot. 96, 461-466. doi: 10.1093/aob/mci197

Liu, C., Song, G., Zhou, Y., Qu, X., Guo, Z., Liu, Z., et al. (2015). OsPRR37 and Ghd7 are the major genes for general combining ability of DTH, PH and SPP in rice. Sci. Rep. 5:12803. doi: 10.1038/srep12803

Liu, Y., Chen, L., Liu, Y., Dai, H., He, J., Kang, H., et al. (2016). Marker assisted pyramiding of two brown planthopper resistance genes, Bph3 and Bph27 (t), into elite rice Cultivars. Rice 9:27. doi: 10.1186/s12284-016-0096-3

Luo, D., Xu, H., Liu, Z., Guo, J., Li, H., Chen, L., et al. (2013). A detrimental mitochondrial-nuclear interaction causes cytoplasmic male sterility in rice. Nat. Genet. 45, 573-577. doi: 10.1038/ng.2570

Luo, X., Fu, Y., Zhang, P., Wu, S., Tian, F., Liu, J., et al. (2009). Additive and over-dominant effects resulting from epistatic loci are the primary genetic basis of heterosis in rice. J. Integr. Plant Biol. 51, 393-408. doi: 10.1111/j.1744-7909.2008.00807.x

Murray, M. G., and Thompson, W. F. (1980). Rapid isolation of high molecular weight plant DNA. Nucleic Acids Res. 8, 4321-4325. doi: 10.1093/nar/8.19.4321

Qiu, Y., Guo, J., Jing, S., Zhu, L., and He, G. (2010). High-resolution mapping of the brown planthopper resistance gene Bph6 in rice and characterizing its resistance in the 9311 and Nipponbare near isogenic backgrounds. Theor. Appl. Genet. 121, 1601-1611. doi: 10.1007/s00122-010-1413-7

Shamsudin, N. A., Swamy, B. P., Ratnam, W., Sta Cruz, M. T., Sandhu, N., Raman, A. K., et al. (2016). Pyramiding of drought yield QTLs into a high quality Malaysian rice cultivar MRQ74 improves yield under reproductive stage drought. Rice 9:21. doi: 10.1186/s12284-016-0093-6

Suh, J. P., Jeung, J. U., Noh, T. H., Cho, Y. C., Park, S. H., Park, H. S., et al. (2013). Development of breeding lines with three pyramided resistance genes that 
confer broad-spectrum bacterial blight resistance and their molecular analysis in rice. Rice 6:5. doi: 10.1186/1939-8433-6-5

Suh, J. P., Yang, S. J., Jeung, J. U., Pamplona, A., Kim, J. J., Lee, J. H., et al. (2011). Development of elite breeding lines conferring Bph18 gene-derived resistance to brown planthopper (BPH) by marker-assisted selection and genome-wide background analysis in japonica rice (Oryza sativa L.). Field Crops Res. 120, 215-222. doi: 10.1016/j.fcr.2010.10.004

Suresh, P. B., Srikanth, B., Kishore, V. H., Rao, I. S., Vemireddy, L. R., Dharika, N., et al. (2012). Fine mapping of Rf3 and Rf4 fertility restorer loci of WA-CMS of rice (Oryza sativa L.) and validation of the developed marker system for identification of restorer lines. Euphytica 187, 421-435. doi: 10.1007/s10681-012-0737-6

Tang, H., Luo, D., Zhou, D., Zhang, Q., Tian, D., Zheng, X., et al. (2014). The rice restorer Rf4 for wild-abortive cytoplasmic male sterility encodes a PPR protein that functions in reduction of WA352 transcripts. Mol. Plant 7, 1497-1500. doi: $10.1093 / \mathrm{mp} / \mathrm{ssu} 047$

Weng, J., Gu, S., Wan, X., Gao, H., Guo, T., Su, N., et al. (2008). Isolation and initial characterization of GW5, a major QTL associated with rice grain width and weight. Cell Res. 18, 1199-1209. doi: 10.1038/cr. 2008.307

Xi, Z. Y., He, F. H., Zeng, R. Z., Zhang, Z. M., Ding, X. H., Li, W. T., et al. (2006). Development of a wide population of chromosome single-segment substitution lines in the genetic background of an elite cultivar of rice (Oryza sativa L.). Genome 49, 476-484. doi: 10.1139/G06-005

Zhao, Y., Huang, J., Wang, Z., Jing, S., Wang, Y., Ouyang, Y., et al. (2016). Allelic diversity in an NLR gene BPH9 enables rice to combat planthopper variation. Proc. Natl. Acad. Sci. U.S.A. 113, 12850-12855. doi: 10.1073/pnas.1614 862113

Zhou, P. H., Tan, Y. F., He, Y. Q., Xu, C. G., and Zhang, Q. (2003). Simultaneous improvement for four quality traits of Zhenshan 97, an elite parent of hybrid rice, by molecular marker-assisted selection. Theor. Appl. Genet. 106, 326-331. doi: $10.1007 / \mathrm{s} 00122-002-1023-0$

Conflict of Interest Statement: The authors declare that the research was conducted in the absence of any commercial or financial relationships that could be construed as a potential conflict of interest.

Copyright (C) 2017 Fan, Li, Chen, Liu, Sun, Wang, He, Zhu and Li. This is an open-access article distributed under the terms of the Creative Commons Attribution License (CC BY). The use, distribution or reproduction in other forums is permitted, provided the original author(s) or licensor are credited and that the original publication in this journal is cited, in accordance with accepted academic practice. No use, distribution or reproduction is permitted which does not comply with these terms. 